Dieses Dokument ist eine Zweitveröffentlichung (Verlagsversion) / This is a self-archiving document (published version):

Alexander Lasch

„Die A[ssassinen] sollen aus Ägypten stammen“

Erstveröffentlichung in / First published in:

Christian Braun, Hrsg. Sprache und Geheimnis. Berlin: Akademie Verlag 2012, S. 89 - 106. ISBN 978-3-05-006012-5.

DOI: https://doi.org/10.1515/9783050060125-006

Diese Version ist verfügbar / This version is available on:

https://nbn-resolving.org/urn:nbn:de:bsz:14-qucosa2-748453 


\section{ALEXANDER LASCH \\ „Die A[ssassinen] sollen aus Ägypten stammen“ \\ - Geschichte(n) eines radikal-islamischen Ordens und ihre Diskursivierung an der Schwelle zur Moderne}

\section{Einleitung}

Die Assassinen stammen nicht aus Ägypten, wie in der Einleitung des Lexikonartikels „Assassinen“ in Pierer's Universal-Lexicon (1857: 830) gemutmaßt wird, sondern sind vom ausgehenden 11. bis ins 13. Jahrhundert der militärische Arm der Ismailiten Persiens und Syriens, der „Siebener-Schia“, die ideologisch und religiös weit außerhalb der sunnitischen Mehrheit des Islams und selbst des schiitischen Islams stand und steht (vgl. Lewis 2001: 39ff.). Die besonderen Umstände der (kurzen) Geschichte dieses Ordens machen darauf aufbauende Darstellungen der Geschichte der Assassinen in unserem Kontext - dem Nachdenken über die Art und den Gegenstand einer Arkanlinguistik - aus drei Gründen besonders interessant.

(1) Die Assassinen versetzten zwischen 1090 und 1273 die islamische Welt und damit auch die „Franken“, die zu dieser Zeit im Heiligen Land sind, in Angst und Schrecken, weil sie gezielt autokratische Machtinhaber ermorden und dabei den eigenen Tod in Kauf nahmen, überaus geschickt, erfolgreich und vor allem loyal gegenüber ihrem Gebieter, dem so genannten „Alten vom Berge“, waren. Mit diesem (in der christlichen und islamischen Welt) als gottlos bewerteten Handeln geben sie in der christlichen Welt schon früh Anlass zu zahlreichen Spekulationen über ihre Beweggründe.

(2) Die Zahl der Berichte über die Assassinen, die ins Abendland kommen, steigt drastisch an, als der Orden berühmt und berüchtigt wird wegen des erfolgreichen Attentats auf den König von Jerusalem, Konrad von Montferrat (*1146) im Jahr 1192 (vgl. Maalouf 2008: 230; Lewis 2001: 160f.). Zwar hatten sich die Nachrichten über die vorangegangenen (erfolglosen) Anschläge auf Sultan Saladin, Salah ad-Din Yusuf bin Ayyub (1137/38-1193, Sultan 1171), die auch dem „Alten“, Sinan ibn Salman ibn Mu- 
hammad, Raschid al-Din (1133/35-1192, Führer der Assassinen in Persien 1164), zugeschrieben wurden, bereits wie ein Lauffeuer in der islamischen und damit auch der christlichen Welt verbreitet - doch die Ermordung Konrads hatte eine neue Qualität. Ungeheuerliches hörten die Christen nun vom „Alten“, doch sie wussten kaum Nennenswertes über ihn oder die Gruppe der Mörder.

(3) Die Assassinen gingen zweimal unter, einmal in der Geschichte und zugleich in den Geschichten. In der zweiten Hälfte des 13. Jahrhunderts verlieren sie zum einen ihre Machtzentren - Alamut in Persien wird durch mongolische Steppenvölker zerstört (1256), ihre syrischen Festungen nimmt bis 1273 der mamelukische Sultan Baibars ein (1223-1277, Sultan 1260). Zum anderen wurde das Wissen über sie verschüttet. Es verbrannte in der Bibliothek von Alamut oder wurde in der islamischen, d. h. der sunnitischen, Welt als sektiererisches nicht tradiert. Nur in wenigen ismailischen Quellen blieben Details über die Geschichte der Assassinen erhalten, hier existierten außerhalb des Fokus der morgenländischen und abendländischen Gelehrten Biographien der Führer der Assassinen, ideologische und theologische Schriften weiter.

Die Voraussetzungen für eine Legendenbildung sind ideal: Wegen ihrer schrecklichen und mit erschreckender Perfektion ausgeführten Taten waren die Assassinen weit über das Morgenland hinaus berühmt und berüchtigt. Die Geschichte und die Struktur des Ordens aber blieben im Dunkeln, über ihre Motivation und ihre Beweggründe wusste man im christlichen Abendland kaum etwas.

\section{Theoretische Vorannahmen}

\subsection{Gegenstände einer Arkanlinguistik}

Unter einem ,Arkanum' versteht man mit dem Blick auf die Etymologie des Wortes etwas Verschlossenes, etwas Nicht-Erfassbares oder Exklusives. ${ }^{1}$ Ausgehend von dieser einfachen etymologischen Bestimmung ist es möglich, (wenigstens) zwei Perspektiven anzunehmen, um eine Arkanlinguistik zu entwerfen und ihren Gegenstand zu bestimmen:

Zum einen schränkt eine enge Bestimmung den Gegenstand einer Arkanlinguistik auf Sprachen bzw. Sprachhandlungen ein, die eingeweihte Benutzer des Idioms oder nicht eingeweihte Beobachter als arkan bezeichnen. Gerhard Eis verwendete in seinen Studien den Begriff Arkansprache genau in diesem Sinne, ohne aber einen Terminus zu entwerfen, für ein Idiom, welches unter Alchemisten dann genutzt wurde, wenn ,geheimzuhaltende Arbeitsmethoden beschrieben wurden“ (vgl. Eis 1965: 64). Diese (meist nur temporär) ,geheimen' Fach-, Sonder- und Gruppensprachen dienen der ge-

${ }^{1}$ Vgl. lat. arcanus, ursprünglich ,verschlossen', im übertragenen Sinne ,verschwiegen', ,geheim‘, ,heimlich'; das Substantiv arcanum meint das ,Geheimnisvolle‘, ,Geheimnis‘ (Georges, Karl Ernst: Lateinisch-Deutsches HandWörterbuch, Bd. 1: Sp. 540). 
schützten, exklusiven Verständigung einer eingeweihten Gruppe in Gegenwart einer nicht eingeweihten Gruppe. Dabei ist das Idiom meist nicht sich selbst Zweck, sondern es dient der Geheimhaltung des kommunizierten Wissens. Für die Bewertung einer Sprache als Arkansprache ist dabei aber weniger relevant, ob das ,Geheimnis' dieser Sprache irgendwann entdeckt wird, d. h., ob sie von nicht eingeweihten Beobachtern entschlüsselt und damit das in ihr kommunizierte Wissen offenbar wird, sondern der selbst behauptete oder von außen wahrgenommene Anspruch einer Gruppe, dieses Wissen als exklusives Wissen mittels eines Idioms verbergen zu wollen.

Zweitens öffnet eine weite Perspektive den Blick für diskursive Praktiken, die Exklusivität als Arkanum verhandeln. Ich werde mich darauf konzentrieren. Auf der Basis soziolinguistischer Annahmen und text- sowie diskurslinguistischer Methoden werde ich am Beispiel der Diskursivierung von Wissen über den Orden der Assassinen herausarbeiten, dass unter Arkana in einem (sozio-)linguistischen Sinn vor allem (stereotype) Zuweisungen von Exklusivität zu fassen sind, die kommunikativ verhandelt werden. Arkana können vor diesem Hintergrund alle komplexen Zeichen und nicht nur Sprache sein (Wissen, Sprache und [Sprach-]Handlungen, Räume, Zeiten, Riten, Gegenstände usw.), die als exklusiv bewertet werden und deren Exklusivität diskursiv verhandelt wird. Soziolinguistisch motiviert würde man dann, eng an das Interesse der Stereotypenforschung angelehnt (vgl. Quasthoff 1973, 179ff.), ${ }^{2}$ Fragen zu stellen haben, wie: Welche Gruppe weist auf welche Art kommunikativ Exklusivität zu? Welche Funktion übernimmt die kommunikative Zuweisung zwischen verschiedenen Gruppen einer Gesellschaft (vgl. Schjerve-Rindler 2004)? Kann man Praxen, Symbolsysteme, Orte und Zeiten, die, wie in unserem Fall, für eine Gruppe als besonders relevant eingeschätzt werden, kommunikativ ,arkanisieren" und welche sprachlichen Strategien werden dazu eingesetzt? Geht mit der ,Arkanisierung ${ }^{6}$ - also der kommunikativen Zuweisung von Exklusivität - eine Bewertung einher und welche Einstellungen werden daran sichtbar (vgl. Strasser / Brömme 2004; Lasagabaster 2004) usf.

\subsection{Transfer und Diskursivierung von Wissen}

Um diesen soziolinguistischen Fragen nachgehen zu können, werde ich mich (1) auf die Erkenntnisse der so genannten „Transferwissenschaften“ stützen, die den Transfer von Wissen modellhaft als Prozesse der Initiierung, des Flusses und der Integration von Wissen verstehen (vgl. Antos / Wichter 2001, 2005; Busch / Stenschke 2004; Stenschke / Wichter 2009 u. a.). Dem Transfer geht die Exploration von Wissen (meist) voraus, beide Prozesse beeinflussen sich mitunter aber auch wechselseitig - der Anschaulichkeit halber werde ich mich hier auf die Exploration und die Integration von Wissen konzentrieren. Das Ergebnis des Zusammenwirkens von (explorierten) Wissensbeständen in der Expertenkommunikation, der Laienkommunikation und auf den Zwischen-

2 Vgl. daneben bspw. Bourhis / Maass (2005); Löffler (2010: 42f.). 
stufen des Transfers lässt sich mit dem Begriff des Diskurses fassen. Dieser Diskurs, der in unserem Fall Arkana als Exklusives kommunikativ verhandelt, lässt sich (2) mittels eines diskursanalytischen Modells beschreiben (vgl. etwa Warnke/Spitzmüller 2008). Im Vordergrund wird aber hier nicht der Diskurs als ,kommunikatives Ergebnis selbst stehen, sondern der Prozess der Diskursivierung: Ich frage nach den diskursprägenden (kommunikativen) Entstehens- und Gelingensbedingungen und Regeln des Diskurses, rücke die transtextuelle Ebene und die Ebene der Akteure eines Diskurses mit in den Blick. Auf diese Weise kann ich am Beispiel der Geschichten über den Orden der Assassinen die Art und Weise der Generierung von Wissen, das Zusammenwirken von Wissensbeständen und vor allem die Rolle von Diskursakteuren, die sowohl horizontal (also auf der Ebene der Expertenkommunikation im Prozess der Wissensexploration und des Transfers) als auch vertikal (in der Experten-Laien-Kommunikation im Transfer) Diskurspositionen vertreten und kommunikativ durchzusetzen suchen.

\section{Wissensexploration: die Darstellung der Assassinen in abendländischen Quellen}

Im Folgenden werde ich am Beispiel der Darstellungen des Ordens der Assassinen den im theoretischen Teil aufgeworfenen Fragen nachgehen, die ich noch einmal kurz zusammenfassen möchte: Wie und was wird über den Orden - soziolinguistisch als Gruppe gefasst - in abendländischen Quellen berichtet? Wird in den Darstellungen Exklusivität verhandelt und wie wird sie bewertet? Welches Wissen also wird auf welche Weise über den Orden transferiert? Welche Diskursakteure sind an diesem Wissenstransfer beteiligt und welche Diskursposition vertreten sie? Sind für den Erfolg einer Diskursposition auch sprachliche Strategien mitverantwortlich? Bei der Beantwortung dieser Fragen werde ich nicht auf generelle Fragen der Islamkritik in Europa eingehen, die in unterschiedlichen Zeiten mit unterschiedlicher Motivation und Schärfe vorgetragen wurde. Bekannt ist, dass selbst Aufklärer Ressentiments gegenüber dem Islam und seinem Stifter hatten und dies auch zum Ausdruck brachten. Dies gilt auch noch für das 19. Jahrhundert; das positive europäische Orientbild und -ideal ist in weiten Teilen ein areligiöses und daher in Fragen der Islamkritik nicht in Anschlag zu bringen. Im Einzelnen werde ich auf diesen Problemkomplex hier nicht eingehen können, möchte aber anmerken, dass dieser Hintergrund bei allem hier Gesagten mitzudenken ist.

Ausgehend von Berichten aus der Kreuzzugszeit werde ich mich in einem zweiten Schritt der Weltchronik Jansen Enikels und einer zwar sehr kurzen, dafür aber sehr einflussreichen Darstellung der Assassinen in Marco Polos Reisebeschreibungen zuwenden (Strauch 1980; Polo 1477). Mein Hauptaugenmerk werde ich auf zwei deutschspra- 
chige Publikationen an der Schwelle zur Moderne richten: ${ }^{3}$ Withof (1765) und Hammer-Purgstall (1818) verfolgen als Diskursakteure je ein klares Ziel in ihrer Betrachtungs- und Darstellungsweise des Bundes. Sie initiieren den Transfer von Wissen zwar nicht, suchen aber wohl den Fluss des Wissens zu steuern, zu kontrollieren und das zu integrierende Wissen zu empfehlen - an verschiedenen Lexikoneinträgen aus dem 18. und 19. Jahrhundert möchte ich in einem letzten Schritt zeigen, welche Diskursposition sich schließlich durchsetzte und die Frage zu beantworten suchen, welche Rolle die kommunikative ,Arkanisierung' einer Gruppe dabei spielte.

\subsection{Kreuzzugszeit}

Beginnen möchte ich mit dem Bericht Gerards, einem Gesandten Friedrich Barbarossas (1122-1190, Kg. 1152, Ks. 1155), der wohl als einer der ersten zwischen dem zweiten und dritten Kreuzzug 1175 Nachrichten über ein „Geschlecht der Assassinen“ in den Westen trägt. In Phönizien, so berichtet er, ${ }^{4}$

wohn[e] ein gewisses Geschlecht der Sarazenen, die in ihrer eigenen Sprache ,Heyssessini“, im Romanischen aber ,segnors de montana' genannt werden. Diese Menschenbrut lebt ohne Gesetz, verzerrt Schweinefleisch [...] und verkehrt unterschiedslos mit allen Weibern, die eigenen Mütter und Schwestern eingeschlossen. [...] Sie gehorchen einem Meister, der alle sarazenischen Fürsten nah und fern in größte Furcht versetzt, ebenso alle christlichen Landesherren. Denn er pflegt auf ungewöhnliche Art und Weise zu töten. Seine Methode ist die folgende: Dieser Herrscher besitzt in den Bergen zahlreiche prächtige Paläste, umgeben von sehr hohen Mauern, die man lediglich durch eine kleine, sehr gut bewachte Tür betreten kann.

Sie lehrten ihre Jungen in den verschiedensten Sprachen und verpflichteten sie zum unbedingten Gehorsam gegenüber dem ,Meister', bis sie schließlich „,vor den Herrscher gerufen werden, um jemanden zu töten. [...] Auf das Gelöbnis hin überreicht der Herrscher jedem von ihnen einen goldenen Dolch und sendet sie aus, den Fürsten zu töten, den er für jeden von ihnen ausersehen hat" (Lübeck 2001: 17) Wilhelm von Tyrus (1130-1186) fügt wenig später hinzu:

Das Band der Ergebenheit und des Gehorsams, das dieses Volk mit seinem Anführer verbindet, ist so stark, daß es keine Aufgabe gibt [...], die nicht jeder von ihnen mit größtem Eifer übernehmen würde, sobald der Anführer es befohlen hat. Gibt es zum Beispiel einen Fürsten, der $\mathrm{Ha} ß$ oder Mißtrauen dieses Volkes auf sich gezogen hat, so überreicht der Anführer einem oder mehreren seiner Gefolgsleute einen Dolch. Wer immer den Befehl erhält, begibt sich sofort auf den Weg, um seine Mission auszuführen. Er bedenkt weder die Folgen der Tat noch die Möglichkeit des Entkommens. Begierig, seine Aufgabe zu erfüllen, setzt er so lange wie nötig alle seine Kräfte ein, bis das Schicksal ihm die Gelegenheit gibt, den Befehl seines Anführers zu

3 Kurz referieren werde ich dabei Silvestre de Sacy (1818. Mémoire sur la Dynastie des Assassins. In: Mémoires de l'Institut Royal 4, 1 ff. Zuerst vorgelegt 1809), auf den von Hammer aufbaut.

4 Alle Beispiele werden, da zum einen der Fokus auf der Konzeptualisierung von Arkana liegt und zum anderen der Verständlichkeit halber, in Übersetzung gegeben. 
vollstrecken. Wir wie auch die Sarazenen nennen dieses Volk ,Assassinen`; aber die Herkunft dieses Namens kennen wir nicht. (Tyrus 2001: 18)

Mit Arnold von Lübeck $(\dagger 1211 / 1214)$ und dem von ihm verfassten Teil der Chronica Slavorum möchte ich diese erste Annäherung schließen.

Ich werde jetzt Dinge über den Alten berichten, die absurd erscheinen, aber durch Aussagen zuverlässiger Zeugen verbrieft sind. Der Alte hat in seinem Land Menschen durch Hexerei derart verwirrt, daß sie weder beten noch an irgendeinen Gott außer ihn selbst glauben. Überdies verlockt er sie auf seltsame Weise mit Hoffnungen und Versprechungen ewiger Freuden, so daß sie lieber sterben als leben wollen. [...] Der höchsten Seligkeit, versichert er, werden jene teilhaftig, die Menschenblut vergießen und als Vergeltung für solche Taten selbst den Tod erleiden. Haben sich einige für einen solchen Tod entschieden [...], so überreicht er selbst ihnen Dolche, die sozusagen dieser Tat geweiht sind. Dann reicht er ihnen einen Gifttrank, der sie in Ekstase und Vergessen stürzt, versetzt sie durch Magie in phantastische Träume voller Freuden und Wonnen [...] und verspricht ihnen den ewigen Besitz all dessen als Belohnung für ihre Taten. (Lübeck 2001: 18f.)

In den abendländischen Chroniken wird am Ende des 12. Jahrhunderts folgendes Wissen über einen Bund der Assassinen exploriert: Es gibt eine Gemeinschaft um einen ,Alten vom Berge'. Deren zum Gehorsam verpflichtete Mitglieder werden als Assassinen bezeichnet - die Herkunft des Namens bleibt allerdings unbekannt. Diese Gemeinschaft lebt außerhalb des Gesetzes (Schari'a) und der religiösen Gebote; ihre Mitglieder praktizierten den Inzest, berauschten sich und mordeten. Der Alte indoktriniere seine Gefolgschaft in Burgen, die von der Außenwelt nicht erreicht werden könnten. Er ,verhexe' sie, versetze sie in Rausch und Ekstase. Einziger Zweck der Gemeinschaft ist die Tötung muslimischer und christlicher Fürsten. Dazu gebrauchen sie einen Dolch und erleiden dabei meist selbst den Tod - verhießen ist ihnen dafür die ewige Teilhabe an allen Freuden und Wonnen (des Paradieses).

Die Berichte dürften, wie eingangs skizziert, zum einen aus einem sehr grundsätzlichen Interesse an dem Bund nach den erfolglosen Anschlägen auf Saladin (1174/1175 und 1176) und der Ermordung Konrads 1192 in Tyrus motiviert sein, für den in verschiedenen Quellen Richard I. Löwenherz (1157-1199, König von England 1189), Saladin und Sinan selbst verantwortlich gemacht werden (vgl. Lewis 2001: 160f.). Zum anderen flossen in die Darstellungen wohl Gerüchte über die ,Aufhebung des Gesetzes (Schari'a)' und ekstatische Ausschweifungen der ,Reinen' in der Mitte der zweiten Hälfte des 12. Jahrhunderts im syrischen Machtbereich Sinans ein, die zwar von sunnitischen Historikern gern Sinan zugesprochen, von ihm selbst aber vielmehr militärisch unterbunden wurden (vgl. Lewis 2001: 152).

\subsection{Spätmittelalter und Frühe Neuzeit}

Es mag seine Ursache im bildmächtigen aber dabei unterspezifizierten explorierten Wissen über die Assassinen haben, dass neben die darstellenden Berichte früh legenda- 
rische Entwürfe über den ,Alten' traten. Wahrscheinlich die erste Aneignung dieser legendarischen Stoffe in einer geschichtlichen Darstellung ist der Bericht über Friedrich II. (1194-1250, 1198 Kg. von Sizilien, 1211/1212 dt. Kg., 1220 Ks.) in Jan Enikels Weltchronik: „Nû merket, keiser Fridrîch, | [...] er hiez stecher ziehen: | an swem er sich wolt rechen, | den hiez er waerlich stechen. | der ein fürst was genant, | dem hiez er tuon den tôt bekannt" (Jansen 1980: 28035; 28040ff.). Alle Wissenselemente, die wir oben als konstitutiv für das Bild der Assassinen in den abendländischen Chroniken herausgearbeitet hatten, kehren in dieser Darstellung bezogen auf Friedrich wieder: Friedrich ließe Kinder , unter der Erde' im falschen Glauben, er sei Gott, erziehen. Sie sahen nie das Licht des Tages und würden erst zu Friedrich gerufen, wenn er einen Mordauftrag erteilte: Nichts wünschten sich die dann jungen Männer so sehr, als im Licht (,,in iuwerm himelrîche" [Jansen 1980: 28085]) zu bleiben, was ihnen der Kaiser im Gegenzug zu ihrem Versprechen, den Mord auszuführen, gewährte - in einer Handschrift (e) wird bereits ein paradiesähnlicher Garten des Kaisers beschrieben, der die Darstellungen der verschlossenen Assassinenfestungen, in deren Innern Wunderliches vorgeht, nun ins Bild setzt: Dieser Heterotopos, um mit Foucault (2005) zu sprechen, ${ }^{5}$ der geheime, verschlossene Garten, über den ein ,Alter', von unwissenden jungen Männern als gottgleich verehrt, verfügt, wird im explorierten Wissen über die Assassinen zum zentralen Element werden und im Wesentlichen deren kommunikative Arkanisierung vorantreiben. Das Buch der Wunder Marco Polos erzählt in einer französischen Bilderhandschrift, welche um 1410 im Auftrag von Johann Ohnefurcht (1371-1419) entstand, nicht nur vom Garten des ,Alten', sondern sie zeigt ihn zugleich bildlich. Eine der Miniaturen stellt ein zentrales Motiv des Reiseberichts dar - den in den Bergen liegenden umgrenzten paradiesischen Garten des ,Alten vom Berg ${ }^{6}$, der nur durch eine Tür begehbar ist und in dem junge Männer die Freuden des Paradieses genießen. Dazu zählen Vergnügungen jeder Art, über die der ,Alte' stehend gebietet. Es ist aber nicht die bildliche Darstellung in einer Bilderhandschrift, sondern der nicht sonderlich umfangreiche Bericht über die Assassinen (oder besser den ,Alten vom Berge') selbst (Polo 1477: 16rff.), der mit dem Fokus auf der Darstellung des Heterotopos ,Garten' eines der wirkmächtigsten Bilder in allen auf ihn folgenden Darstellungen über den Bund der Assassinen aufruft. Auch wenn das über die Assassinen explorierte Wissen im Detail erweitert wird - die Gemeinschaft sei eine muslimische, der Alte jedoch nutze die Religion gänzlich eigennützig und verbrecherisch aus, wozu er auch seinen Garten gebraucht -, so ist doch die Konzentration auf den Heterotopos bereits in der Überschrift des Kapitels augenscheinlich: „Von dem scho̊nstē uñ wunderlichstē gartē der welt den [der] veglio vō perge gemacht hett | uñ sprach es wer das paradeyß“" (Polo 1477: 16r). Sieht man von der marginalen Erwähnung in Enikels Chronik ab, so war den Außenstehenden bisher das Innere der Festen der Assassinen verborgen, nun öffnet der Bericht Marco Polos den Blick auf einen Garten, den der ,Alte` selbst das ,Paradies' nennt:

5 Gemeint sind räumlich lokalisierbare Gegenräume, die „stets ein System der Öffnung und Abschließung besitzen, welches sie von der Umgebung isoliert“" (Foucault 2005:18). 
Dar em hat er lassen machen den schónstē garten des gleich en von keynem menschen nye meer gesehen wardt $\mid$ vnd dar inn die edelstē frücht die von keynem menschen nye gesehen wurden. Auch den schónsten pallast von allem lust gezirtt mit golde vnd mit edelem gesteyn. In dem garten vmb den pallast seyn vil scho̊ne prunnen. Etlich geben weyn | etlich wasser | auch seyn prunnen dy da milch geben. Dar innen waren die schónsten frawen und Junckfrawen die man ye gesehen hat. (Polo 1477: 16r)

Der ,Alte‘ lässt junge Männer trickreich - er verabreicht einen Schlaftrunk, um sie in den Garten hinein- und hinausbringen zu lassen - an den Freuden des Paradieses in seinem Garten teilhaben und verspricht ihnen als Lohn, dass sie in dieses Paradies eingehen werden:

er sprach alle die da storben in seinem gepot vin untertenikeyt das die alle engel in dē paradeyß wurdē $\mid$ also er plēdet das volck vn̄ vil grosser her[ren] die sorg vñ forcht vor im hettē $\mid$ vñ reychten im ein genantes gelt alle iar. (Polo 1477: 16v)

Dieser Heterotopos, in dem die Regeln des Alltags aufgehoben sind zugunsten eines von außen nicht sichtbaren paradiesischen Lebens, wird, wie im Folgenden zu zeigen sein wird, die Exploration von Wissen und den Transfer des Wissens über die Assassinen aus der Expertenkommunikation in die Laienkommunikation maßgeblich prägen: Das Wissen über den als Arkanum eingestuften und bewerteten Garten wird integrierbares Wissen über die Assassinen. Mit der Darstellung des Heterotopos des Paradiesgartens erhält das explorierte Wissen über den Alten und seine Assassinen so einen neuen Kern. Das Bildensemble wird wesentlich erweitert: Neben dem Garten sind es die unbezwingbaren Festen, der Absolutheitsanspruch des die Religion des Islam ausnutzenden (und daher gott- und gesetzlosen) betrügerischen Alten und der absolute Gehorsam der Assassinen mit ihren geweihten Dolchen, die Fürsten töten - und im Auftrag ihres Herrn räuberisch erpressen.

Paradoxerweise, und das klang bereits in der Einleitung und soeben an, wird mit dem Garten zum einen das Arkanum genannt, es wird gezeigt und damit seiner Exklusivität entledigt -weder in Wort und Schrift noch im Bild ist der Garten des Alten mehr arkan. Zum anderen aber wird das Gartenmotiv als beschriebener Heterotopos zum tragenden Element im Diskurs über die Assassinen und damit der kommunikativen Arkanisierung des Ordens.

\subsection{Schwelle zur Moderne}

1765 veröffentlicht Joh. Phil. Lorenz Withof seine Darstellung des meuchelmoerderische $[n]$ Reich $[s]$ der Assassinen. Die Monographie spiegelt den Wissensstand europäischer Historiker in der Mitte des 18. Jahrhunderts wider und ist ganz im Sinne der Aufklärung verfasst. Withof übt bereits in der Einleitung Kritik sowohl an religiösem Fanatismus wie der eurozentrischen Perspektive in geschichtlichen Darstellungen: 
Um dessentwillen ließe sie [die Vorsicht, A.L.] es auch geschehen, daß die Europ[ae]r mit ihrem Aberglauben [dem christlichen Glauben, A.L.] nach Asien reiseten. Sie tummelten sich da eine Zeitlang gewaltig herum. Die Mahometaner jagten sie endlich wieder nach Hause. Sie setzten sich abermals im Schooße des Aberglaubens ruhig hin, und fielen von neuem in Schlaf. (Withof 1765: 5)

Withof ist an einer historisch-wissenschaftlichen Arbeit nach modernem Verständnis über die Assassinen interessiert, wie er im Vorbericht ausführlich darlegt. Sein Anliegen als Diskursakteur ist es, eine moderne Darstellung des Ordens der Assassinen im Lichte der Aufklärung ohne religiöse und kulturelle Vorurteile vorzulegen. Seine Studie erweitert das Wissen über die Assassinen wesentlich und gibt auf zahlreiche Fragen Antworten. Der Führer der Assassinen werde als Scheykh bezeichnet, was nichts anderes als ,Fürst' oder ,Alter' im Sinne des, Ältesten' bedeute (Withof 1765: 30f.). Die Bezeichnung des Ordens, dessen Namen auch er nicht klären kann, als ,Sekte‘ sei zumindest missverständlich, diese Charakterisierung diente den Chronisten der Kreuzzüge, verschleiere aber das Wesen des Bundes (Withof 1765: 10). Vielmehr seien die Assassinen der ismailischen Lehre verpflichtet, die zur Schia gehört. Withof deckt das „geheime Gesetz der Assassinen“ auf (Withof 1765: 49ff.) - eine Folge von Initiationsschwüren - und stellt die Struktur der Gemeinschaft ausführlich dar: Die Assassinen gliederten sich in Untertanen, eine Armee und die ,Garde'. Dies sind die ,Weißen', die im Auftrag des ,Alten' Anschläge verübten. Armee und Garde war dabei gemein,

daß sie in vollkomner Einigkeit lebten; keine Ehrsucht kennten; die Befơrderung ihres Glaubens für ihr gemeines Beste achteten; für ihr gemeines Beste dem Tode sehnlich entgegen giengen; sich eine besondre mahometanische Heiligkeit anmaßten; die Ermordung ihrer gemeinschaftlichen Feinde, voraus aber den anbefohlnen Selbstmord, für das sicherste Unterpfand des Himmels ansahen; und endlich einen blinden Gehorsam gegen ihr Oberhaupt für den achten Artikel ihres geheimen Gesetzes hielten. (Withof 1765: 64)

Auch die Motivation für die Anschläge arbeitet Withof heraus: Die Assassinen verteidigen ihre ismailische Lehre gegen die sunnitische Mehrheit des Islams und seine Repräsentanten, die die Ismailiten (auch militärisch) verfolgten - sie sind als ein radikaler islamischer Orden einzustufen, wie es ebensolche unter den Christen gab. Zusammenfassend vergleicht Withof folgerichtig die Assassinen mit dem Orden der Tempelritter. Beide hätten ihre Hoch- und Blütezeit etwa 180 Jahre während der Kreuzzüge von 1090 bis 1271 erlebt und ,[b]eyde hatten von dem, was sie Religion nannten, in der That einerley Begriff, und hielten die Ermordung desjenigen, der auf eine andre Weise sich gegen Gott verhălt, fứr eine grosse Frómmigkeit.“ (Withof 1765: 181f.) Damit bekräftigt er im Schluss sein Urteil über die Assassinen, das er bereits als These an den Beginn seiner Publikation gesetzt hatte: „[D]ie Assassinen [müssen] ursprünglich für nicht viel mehr, als für eine Gattung mahometanischer Ordensritter gehalten werden" (Withof 1765: 9).

Zwei wesentliche Aspekte kann Withof nicht klären. Er deckt zum einen die Bedeutung des Namens der Assassinen nicht auf und er entlarvt zum anderen nicht den Gartenmythos. Den Kern des explorierten Wissens geht Withof, der als erster in vielen 
Aspekten einen unverstellten Blick auf die Assassinen wirft und zahlreiche Vorurteile und Stereotype bei Seite schiebt, nicht an. Er gibt die Schilderungen des Paradiesgartens Marco Polos wieder, erläutert den Aufbau der Anlagen und vermerkt in einer Anmerkung, dass wohl ,nicht beståndig [Milch] in diesen Båchen geflossen habe, sondern nur nach Belieben des Sheykhs hineingelassen worden sey. Im Orient waren dergleichen Båche recht nach dem herrschenden Geschmacke." (Withof 1765: 39 mit Anm. 51). Mit diesem Kommentar, der den Mythos zu entzaubern sucht aber zugleich kaum glaubhaft voraussetzt, dass Withof von der Existenz solcher Apparaturen am Ende des 12. Jahrhunderts ausging, entkräftet der Aufklärer den Gartenmythos nicht, sondern schreibt ihn weiter in der Diskurstradition fort.

Aufklärerischen Geist atmet auch die Arbeit Mémoire sur la Dynastie des Assassins von Silvestre de Sacy, die er erstmals 1809 in Paris vorstellt und die 1818 veröffentlicht wurde. De Sacy wertet bisher im Westen unbekannte arabische Quellen der Kreuzzugszeit, die Napoleons Truppen in Nordafrika erbeuteten und nach Frankreich brachten, aus und kommt besonders in der Frage nach der Herkunft des Namens der Assassinen einen großen Schritt voran. Er leitet den Namen von arabisch haschissch, bekannt in den Formen haschīschī und haschschāsch ab. Sektierer, so de Sacy, werden durchgehend als haschīschī bezeichnet, nicht als haschschāsch, ein jüngeres Wort, mit dem üblicherweise Haschischkonsumenten gemeint seien. De Sacy schließt daraus, dass für die ,Assassinen` Haschischkonsum zwar nicht üblich war, sondern vermutet, dass Haschisch als Rauschmittel geheim eingesetzt wurde durch den ,Alten“ (vgl. Lewis 2001: 27ff.). Wie wir heute wissen, ist dieser Schluss de Sacys zu korrigieren:

Der Ausdruck haschīschī war auf Syrien beschränkt und wahrscheinlich eine populäre Beschimpfung. Aller Wahrscheinlichkeit nach war es eher dieser Name, der die Geschichte [von einer drogenabhängigen Sekte im Paradiesgarten des Alten] entstehen ließ, als umgekehrt. Von diversen Erklärungsmodellen, die angeboten wurden, ist das nächstliegende, daß es sich um einen Ausdruck der Verachtung für die phantastischen Glaubensinhalte und das extravagante Benehmen der Sektierer handelte - einen höhnischen Kommentar zur ihrer Aufführung eher denn eine Beschreibung ihrer Praktiken. (Lewis 2001: 29)

Am Beginn des 19. Jahrhunderts allerdings liefert de Sacy den Schlüssel, der den Kern des explorierten Wissens, den Paradiesgarten, und die Praxis des ,Alten', junge Männer zu berauschen, um sie in den Garten hinein- und wieder herausbringen zu lassen, bestätigt: Die Mitglieder des Ordens heißen nach dem Rauschmittel, dessen sich der ,Alte“ bediente. Damit stellt de Sacy - wenn auch vorsichtig - die Legende vom Paradiesgarten auf ein etymologisches Fundament.

Joseph von Hammer-Purgstall legt wenig später Die Geschichte der Assassinen aus morgenländischen Quellen (1818) vor, die „anderthalb Jahrhunderte lang die Hauptgrundlage für das populäre Bild von den Assassinen im Westen“ (Lewis 2001: 30) war. Der aufklärerische Impetus Withofs ist nur noch in Ansätzen zu erkennen, nämlich dann, wenn sich von Hammer-Purgstall gegen den Missbrauch der Religion durch die Politik wendet: „Religions-Fanatismus wird von der Geschichte vielfältig als Urheber 
blutiger Kriege [...] angeklagt; doch war die Religion niemals das Ziel, sondern meistens nur Werkzeug ehrgeiziger Politik und ungezähmter Herrschsucht" (HammerPurgstall 2008: 19). Als Diskursakteur ist es Hammer-Purgstall aber zentrales Anliegen, sich - vor dem Hintergrund der Erfahrung des Terrors in Folge der Französischen Revolution - gegen (aufklärerische Toleranz und) gewaltbereite und -tätige Gemeinschaften zu stellen, die die hergebrachte (monarchische) Ordnung gefährden:

Der politische Wahnsinn der Aufklärer, welche die Völker mündig, dem Schirmbund der Fürsten und dem Gängelband positiver Religion entwachsen glaubten, hat sich wie unter der Regierung des Großmeisters Hassan II. in Asien so in Europa durch die Wirkungen der französischen Revolution auf das persönlichste kundgegeben, und wie damals die Lehre des Meuchelmords und aller staatsauflösenden Verbrechen von Alamut offen ausging, so die Lehre des Königsmordes aus dem französischen Nationalkonvent [...]. Die Mitglieder der Konvention [...] wären würdige Satelliten des Alten vom Berge gewesen. (Hammer-Purgstall 2008: 142)

Ganz in diesem Sinne stellt von Hammer-Purgstall die Geschichte der Assassinen als Exempel in Analogie zu Templern, Jesuiten, Illuminaten und Freimaurern dar, denn sie alle treffen in ,dem letzten Zweck, alle Könige und Priester überflüssig zu machen, auf einem Punkt wirklich ganz überraschend zusammen" (Hammer-Purgstall 2008: 141).

Die Geschichte der Assassinen hat man sich im Detail als eine Synopse des explorierten Wissens über den Orden der Assassinen vorzustellen. Unser Hauptaugenmerk wird auf dem Umgang mit der Gartenlegende Marco Polos und der bei de Sacy vorgeschlagenen Lesart des Namens der Assassinen liegen. Im Gegensatz zu Withof und de Sacy pflegt von Hammer-Purgstall allerdings nicht, vorsichtig zu differenzieren und vor möglichen Fehlinterpretationen zu warnen, sondern geht sowohl in der Übersetzung als auch in der Spekulation weiter, ohne dies in der Darstellung zu kennzeichnen: Ihm ist an einer Dichotomie gelegen, in der der Orden der Assassinen möglichst alle negativen Bewertungen eindeutig auf sich vereint. Von Hammer-Purgstall bestätigt - im Nachgang der Lektüre und „umständlichen Auseinandersetzung“ mit de Sacy - die unzweifelhafte Glaubwürdigkeit Marco Polos mit dem recht fragwürdigen Argument der Stabilität der Überlieferung der Geschichte, das uns aber einen wichtigen Hinweis auf die Gelingens- und Entstehensbedingungen des Diskurses gibt, an dem von HammerPurgstall als Akteur mitwirkt:

Seitdem aber dieselbe Beschreibung sich ganz übereinstimmend auch in orientalischen Quellen vorgefunden [...], erhielt Marco Polos Erzählung neue Ansehen, und nachdem seine Wahrhaftigkeit, wie die Herodots, durch ungläubige Jahrhunderte lange bezweifelt worden, erscheint durch die einhellige Aussage orientalischer Geschichts- und Reisebeschreiber die Glaubwürdigkeit des Vaters der alten Geschichte und des Vaters der neuen Reisebeschreibung von Tag zu Tag in immer hellerem Licht. (Hammer-Purgstall 2008: 91)

In seiner Wiedergabe der Erzählung schafft er über seine Vorläufer hinausgehend, wie bei der Bestätigung der „Wahrhaftigkeit“ des Marco Polo, Tatsachen: „Den Jüngling [...] lud der Großmeister / Großprior zu Tisch und zum Gespräch ein, berauschte ihn mit einem Opiat aus Hyoscyamus (Haschisch), und ließ ihn in den Garten tragen [...].“ 
(Hammer-Purgstall 2008: 91) Weiter mutmaßt er, dass wohl nicht nur der ,Alte 'sich des Betrugs mittels der Rauschmittel bediente, sondern über kurz oder lang der Drogenkonsum allen Mitgliedern des Ordens möglich gemacht wurde - die Folgen könne man noch heute in Konstantinopel oder Kairo sehen:

Was bisher als Mittel zum Vergnügen gedient, wurde nun selbst Zweck, und die Begeisterung des Opiumrausches wurde Surrogat himmlischer Freuden [...] und erklärt die Wut, womit jene Jünglinge den Genuss dieser berauschenden Kräuterpastillen (Haschische) suchten, und worin sie durch dieselben versetzt, fähig wurden, alles zu unternehmen. Von dem Genuss derselben nannte man sie Haschischin, das heißt die Kräutler, woraus in dem Mund der Griechen und Kreuzfahrer der Name Assassinen entstanden, der als gleichbedeutend mit Meuchelmörder die Geschichte des Ordens in allen europäischen Sprachen verewigt. (Hammer-Purgstall 2008: 92)

Das explorierte Wissen über die Assassinen umfasst, inklusive der negativen Bewertungen von Hammer-Purgstalls, am Beginn des 19. Jahrhunderts folgende Elemente: Die Assassinen sind eine geordnete und strukturierte Gemeinschaft, in der sieben Grade unterschieden werden, die Attentäter werden als fidāwi (,die Geweihten') bezeichnet. Die Gemeinschaft ist der ismailischen Lehre verpflichtet, die zur Schia gehört. Sie dient einem Sheykh, dem ,Alten', in absolutem Gehorsam. Der ,Alte' indoktriniert junge Männer in von hohen Mauern geschützten Palästen in den Bergen, die nur durch eine kleine Tür zur Außenwelt geöffnet sind. Die Paläste sind von einer paradiesischen Anlage umgeben, in denen die strengen Regeln des alltäglichen Lebens aufgehoben sind - der ,Alte' gebietet über diesen Garten. Die Herkunft und Bedeutung des Namens der Assassinen werden etymologisch richtig hergeleitet, aber zu falschen Schlüssen herangezogen: Der ,Alte“ verführt die Männer in diesem Garten - damit wird die „Wahrhaftigkeit" der Legende postuliert - zum Drogenkonsum, der der Motor für ihre verbrecherischen Handlungen wird. Die gefürchteten Mörder gehören zur Garde (,die Weißen'): Sie geloben dem ,Alten“ die Treue (,geheimes Gesetz der Assassinen“), töten mit einem geweihten, goldenen Dolch und sind selbst bereit zu sterben. Die Gemeinschaft schaltet muslimische und christliche Fürsten aus, die ihre Existenz (und damit den Fortbestand ihrer falschen Lehre) bedrohen. Sie sind als ein radikaler islamischer Orden einzustufen und gefährdeten die Ordnung ebenso wie andere Geheimbünde nach ihnen.

\section{Wissensintegration: das Bild der Assassinen in populären Lexika des 18. und 19. Jahrhunderts}

An Beispielen für die Integration des Wissens über den Orden der Assassinen mangelt es nicht, allerdings wirkt die Publikation von Hammer-Purgstalls 1818 so stark nach, dass ich mich auf zwei Lexikonartikel aus dem langen 19. Jahrhundert beschränken möchte, welchen ich den Eintrag aus Zedlers „Universal-Lexicon“ aus der Mitte des 18. Jahrhunderts voranstellen möchte. Zedlers Artikel steht exemplarisch für den Stand des integrierten Wissens über die Assassinen in der Mitte des 18. Jahrhunderts. Allerdings 
- und hierfür ist das Modell der Transfers von Wissen notwendig - wurden nicht alle Wissenselemente aus der Experten- in die Laienkommunikation transferiert und das schließlich integrierte Wissen ist nur ein (dichotomisch geordneter) Ausschnitt explorierten Wissens, wie es bei Withof entwickelt ist: Die Assassinen - die nie bei ihrem Namen genannt werden, sondern als „Canaille“, „Meuchelmörder“ oder „schändliche Rotte" bezeichnet sind - seien ein Volk in Phönizien mit Burgen in der Gegend von Tyros (Kenntnis hat der Autor wohl nur von den syrischen Assassinen), in denen paradiesische Gärten hinter hohen Mauern lägen, die Organisation gliche einem „Mahometanische[n] Ritter-Orden“, der von einem König geführt würde, der der „Alte des Gebirges“" hieße. Hier würden junge Männer erzogen, die dem ,Alten“ zu absolutem Gehorsam verpflichtet seien und im Auftrag der Gemeinschaft mordeten und raubten (vgl. Zedler 1751: Sp. 1894ff.). Auffällig ist, dass dem Artikel die differenzierende aufklärerische Haltung abgeht, wie wir sie für die Monographie Withofs beschrieben, und damit eine pejorative Bewertung des Ordens möglich wird.

In der Mitte des 19. Jahrhunderts und nach der Publikation von Hammer-Purgstalls werden weitere Wissenselemente aus der Expertenkommunikation diskursiv transferiert und integriert. So ist bspw. im knappen Artikel „Ismaeliten“ im „Brockhaus BilderConversations-Lexikon“ von 1838 der Ursprung der „Sekte“ ins persische Alamut gelegt, der Gründer wird mit „Hassan ben Sabbach el Homairi“ genannt; auch weiß der Artikel einige Informationen zur Geschichte und „Zerschlagung“ der Assassinen zu berichten und über ihr, Wüthendes Verfolgungssystem' gegen die Feinde:

Um in offenem Kampfe aufzutreten, fehlte es ihr [der Sekte] an Macht, daher nahm sie zum Morde, geheimem und öffentlichem, ihre Zuflucht. Es wurden nämlich Mörder förmlich erzogen und abgerichtet, welche, um das ihnen bezeichnete Opfer zu erreichen, keine sie selbst bedrohende Gefahr scheuten. Sie hießen Fedâis, d. h. sich Opfernde, standen unter dem Befehle des Beherrschers der Sekte im Schlosse Alamût, welcher der Alte vom Berge genannt wurde, und berauschten sich, um sich zu ihrem schrecklichen Geschäfte zu ermuthigen, mit einem aus Bilsenkraut bereiteten Getränk. Von dem Namen des Bilsenkrauts wurde die ganze Sekte Haschischi genannt, woraus die Abendländer Assassinen machten. (Brockhaus 1838: 465)

Der Name der Assassinen wird hier in direkte Verbindung zum Drogenkonsum und der daraus resultierenden Gleichmütigkeit gegenüber dem „schrecklichen Geschäfte“ gesetzt. Wie wir oben bei von Hammer-Purgstall sahen, ist dies zwar ein falscher Schluss, aber die daraus begründete negative Bewertung des Ordens ist - wenn schon falsch argumentativ zumindest abgesichert. Ähnlich stellt sich dies auch im Artikel „Assassīnen“ in „Meyers Großem Konversations-Lexikon“ von 1905 dar. Er ist hinsichtlich der Darstellung der Struktur des Ordens der Assassinen hinreichend differenzierend, beutet jedoch argumentativ die Gartenlegende, den Namen der Assassinen, den blinden Gehorsam der Assassinen und deren blutige Taten, um zu einer negativen Bewertung zu kommen, so aus, wie es für die Expertenkommunikation mit von Hammer-Purgstall kennzeichnend war. Durch die Kürze des Artikels tritt die besondere Schärfe der Bewertung deutlich heraus: 
Der Orden zerfiel in mehrere Grade; an der Spitze stand der Scheich ul Dschibâl, was die Abendländer mit Vetulus de montanis oder der Alte vom Berge übersetzten. Lehre und Organisation waren die der Ismaeliten; nur hatte Hassan, um die Unterwürfigkeit der Genossen unter die Obern zu blindem Gehorsam zu steigern, ein teuflisches Mittel ersonnen. Aus den Blättern der Haschischpflanze (des Hauses) wurde nämlich ein starker Trank bereitet, um damit die Jünglinge zu betäuben, die in diesem Zustand an einen Ort, wo alle Reize des Sinnengenusses ihrer warteten, gebracht, nach wenigen Tagen aber auf dieselbe Weise wieder von dort entfernt worden sein sollen. Sie glaubten dann bereits die Freuden des Paradieses genossen zu haben, und, von Sehnsucht nach ähnlichen Genüssen getrieben, gaben sie gern ihr irdisches Dasein dahin. So waren sie die willenlosen Werkzeuge ihrer Obern und verübten jede blutige Tat auf deren Befehl. Als »Hanfesser« nannte man den Fidawi auch Haschschâschi; daraus haben die Franken Assassin gemacht. (Meyer 1905: 888)

Allen drei Artikeln ist gemein, dass sie auf zentrale Elemente des explorierten Wissens zurückgreifen und diese argumentativ so miteinander verknüpfen, dass eine eindeutige Bewertung des Ordens der Assassinen möglich wird. Der (1) Herrschaftsraum der Assassinen, die in Festen leben, wird meist kurz beschrieben und (2) auf die Struktur der Gemeinschaft eingegangen. Dann wird der (3) unbedingte Gehorsam gegenüber dem ,Alten“ hervorgehoben, in dessen Namen (4) die Assassinen Morde und Anschläge begehen. Sie gehorchen ihm, weil er sie (5) in einem paradiesähnlichen Garten mit falschen Versprechungen verführt und berauscht. Dazu wird (6) Haschisch gebraucht, von dem sie auch ihren Namen haben.

\section{Ergebnisse und Ausblick}

Die Gartenlegende ist das stabile Kernelement im explorierten Wissen über die Assassinen an der Schwelle zur Moderne. Mittels der teils falschen aber damit dann im Diskurs eindeutigen Herleitung des Namens der Assassinen und der Stabilität der Überlieferung, die durch eine lange Reihe an Gewährsmännern abgesichert ist, wird die Legende im Transfer argumentativ beglaubigt: Die Assassinen sind eine Gemeinschaft von bezahlten Meuchelmördern, die sich in paradiesischen Gärten berauschen. Während man alle Arkana des Ordens, wie etwa die Hierarchie der Gemeinschaft oder die Initiationsschwüre, aufdeckt, wird auf der anderen Seite mittels des Aus- und Umbaus der Gartenlegende ein Heterotopos - dessen Merkmale Abgeschlossenheit und Exklusivität bei Zugangsbeschränkung sind - diskursiv geschaffen, der dazu dient, der Gruppe der Assassinen einen exklusiven Raum und damit ein Arkanum zuzuweisen. Irrelevant ist dabei, dass dieser Garten in der Realität nicht einmal existiert, bedeutsam aus unserer Perspektive ist, dass mittels der Ausbeutung der Gartenlegende (stereotype und wie in unserem Falle negative) Bewertungen einhergehen. Die Frage nach der Motivation für die Ordensgründung und die Tötung von muslimischen wie christlichen Fürsten, die Schlüsselpositionen im Kampf gegen die ismailische Sekte einnehmen, bleibt hingegen lange Zeit im Dunkeln - im integrierten Wissen spielt dieses Element beinahe keine Rolle. 
Aus den Beobachtungen möchte ich ausgehend von den anfangs formulierten Fragen in Bezug auf den Gegenstand einer Arkanlinguistik hinsichtlich der kommunikativen Arkanisierung $-\mathrm{d}$.h. der Zuweisung von Exklusivität - folgende Schlüsse ziehen und erläuterrn. (1) Das Arkanum ist zu beschreiben als kommunikativ ausgehandelte Exklusivität. Dabei ist es - wie im Beispiel der Geschichte(n) des Ordens der Assassinen deutlich wurde - irrelevant, ob das Arkanum in der Realität existiert oder diskursiv geschaffen wird. (2) Das Wissen über Gruppen und deren Arkana wird mittels besonderer Legitimierungsstrategien beglaubigt. Im vorgestellten Diskurs wurden etymologische Herleitungen und die Stabilität der Überlieferung als argumentative Stützen in Anschlag gebracht. Das explorierte Wissen ist in diesem konkreten Falle als eine Sammlung von „Tatsachen des Bewusstseins“ (Betrand Russell) zu charakterisieren; beglaubigt von einer langen Reihe von Beobachtern zweiter und dritter Ordnung, deren Authentizität dadurch bestätigt wird. Das einzige, was jedoch bestätigt wird, ist das Interesse einer Gruppe an der kommunikativen Arkanisierung einer anderen Gruppe. (3) Das Arkanum selbst wird (im Falle der Assassinen) eindeutig negativ gewertet, da die Gemeinschaft zum einen (im 12. sowie im 19. Jahrhundert) in der Gesellschaft keinen Rückhalt hat und man zum anderen wegen der kausal mit dem Drogenkonsum in Verbindung gebrachten Morde der Gemeinschaft mittels stereotyper Zuweisungen unterstellt, im Verborgenen Tabus zu brechen und Eliten zu bilden, die sich dem Werte- und Normenkonsens der Gesellschaft entziehen. (4) Folge ist dann, dass die einmal stigmatisierte Gruppe Ziel wildester Spekulationen und Anschuldigungen wird. Deswegen werden (5) ,Arkana' einer Gruppe, auch wenn sie längst ihrer Exklusivität entrissen worden sind, dann diskursiv gepflegt, oder neue Arkana etabliert (kommunikative Arkanisierung), wenn das Bedrohungspotenzial der Gruppe für die Gesellschaft aufrecht erhalten werden soll.

Wie wir gesehen haben, setzt sich der Diskursakteur von Hammer-Purgstall gegen Withof sowohl in der Expertenkommunikation als auch im Bereich der Laienkommunikation durch, wenn man die Elemente des integrierten Wissens näher betrachtet, die aus dem explorierten Wissen transferiert worden sind. Auf die Frage, warum dies so ist, kann man im Hinblick auf den hier diskutierten Problembereich verschiedene Antworten geben und neue Fragen stellen, ich möchte mich auf die Passgenauigkeit der sprachlichen Strategien und der Merkmale des Kernelements des explorierten Wissens, den Heterotopos des Gartens, konzentrieren. Der diskursiv geschaffene Heterotopos steht ikonisch für die Abgeschlossenheit und Exklusivität einer Gemeinschaft wie der Assassinen, die mittels der Prozessierung des Heterotopos in der Tradition zunehmend kommunikativ arkanisiert, damit in diesem konkreten Fall negativ bewertet und stigmatisiert werden. Sprachlich versprechen für die Durchsetzung der an einer solchen Bewertung interessierten Diskursposition die Strategien Erfolg, die eine der Basisdichotomien, die der Heterotopos als wesentliches Merkmal aufweist, wie die des Raumes (offen vs. verschlossen), aufgreifen. Weiter können argumentativ andere deiktische Dichotomien ausgenutzt werden, die sich zu dieser Basisdichotomie analog verhalten (bspw. Zeit, 
usuelle Codes [Sprache, Ritus, Kleidung] usw.). Schließlich können ergänzend auch Dichotomien mit evaluativem Charakter (etwa um die Komplexe Wissen, Ordnung, Norm und Tabubruch etc.), die nur noch im übertragenen Sinne als deiktisch verstanden werden können, dazu dienen, das explorierte Wissen in positiv wie negativ bewertete Elemente zu klassifizieren. Für den Wissenstransfer erweisen sich eben die Darstellungen als erfolgreich, die diesen Dichotomien folgen oder sie gezielt aufbauen (von Hammer-Purgstall). Beschreibungen, die auf diese klare Zeichnung und Wertung zugunsten eines neutralen Urteils verzichten (Withof), setzen sich nicht durch - ebenso wenig wie die Diskursposition der jeweiligen Akteure.

Wie an dem hier entwickelten Beispiel der Geschichte(n) des Ordens der Assassinen gezeigt, ist von einer ,Arkanlinguistik ${ }^{\star}$ mit einem weiten Verständnis ,linguistischer Arkana $^{\prime} \mathrm{zu}$ erhoffen, dass die Bedingungen für eine intuitive Bestimmung eines Dingens, eines Raumes, einer Zeit, eines Wissens, einer Person, einer Handlung, eines Ritus usw. als ,Arkanum' präziser beschrieben und methodisch genauer reflektiert werden können. Sonst stünde bei solchen Bestimmungen nämlich durchaus zu befürchten, dass man von Fall zu Fall den Ergebnissen oder den noch andauernden Prozessen kommunikativer Arkanisierungen aufsitzt, die Generationen vor uns mühsam als Pfade durch explorierte Wissensbestände getreten haben.

\section{Literaturverzeichnis}

\section{Quellen}

Brockhaus (Hrsg.) (1809), Brockhaus Conversations-Lexikon. Bd. 7, Artikel Assassinen, Amsterdam, $63 \mathrm{f}$.

Brockhaus (Hrsg.) (1838), Brockhaus Bilder-Conversations-Lexikon. Bd. 2, Artikel Ismaeliten, Leipzig, 465.

Hammer-Purgstall, Joseph von (1818), Geschichte der Assassinen aus morgenländischen Quellen, Stuttgart, Tübingen. [Nachdruck Leipzig 2008].

[Jansen] = Jans(en) (der Enikel) (1980), Jansen Enikels Werke. Weltchronik - Fürstenbuch. Herausgegeben von Philipp Strauch. Nachdr. der Ausgabe 1900, (MGH, Deutsche Chroniken 3), München.

Lübeck, Arnold von (2001), „Chronica Slavorum. IV,16. Übers.“, in: Bernard Lewis, Die Assassinen. Zur Tradition des religiösen Mordes im radikalen Islam, (Die andere Bibliothek 59), Frankfurt am Main, 18f.

Lübeck, Arnold von (2001), „Chronica Slavorum VII, 8. Übers.“, in: Bernard Lewis, Die Assassinen. Zur Tradition des religiösen Mordes im radikalen Islam, (Die andere Bibliothek 59), Frankfurt am Main, 17.

Meyer, Joseph (Hrsg.) (1905), Meyers großes Konversations-Lexikon. Bd. 1, Artikel Assassinen (Assassiden, Assaniten, Hassesinen), Leipzig, 888.

Pierer, Heinrich August (Hrsg.) (1857), Pierer's Universal-Lexikon. Bd. 1, Artikel Assassinen (Assassiden), Altenburg, 830f. 
Polo, Marco (1477), Hie hebt sich an das puch des edeln Ritters vnd landtfarers Marcho Polo, in dem er schreibt die grossen wunderlichen ding dieser welt, Nürnberg. [Bayerische Staatsblibliothek München, 2 Inc.c.a. 652 c.]

Sacy, Silvestre de (1818), „Mémoire sur la Dynastie des Assassins“, in: Mémoires de l'Institut Royal 4, $1 \mathrm{ff}$.

Tyrus, Wilhelm von (2001), „Historia rerum in partibus transmarinis gestarum. XX,31. Übers.“ in: Bernard Lewis, Die Assassinen. Zur Tradition des religiösen Mordes im radikalen Islam, (Die andere Bibliothek 59), Frankfurt am Main, 18.

Withof, Johann Philipp Lorenz (1765), Das meuchelmoerderische Reich der Assassinen. Cleve.

Zedler, Johann Heinrich (Hrsg.) (1732), Großes und vollständiges Universal-Lexicon aller Wissenschaften und Künste. 64 Bde. und 4 Supplement-Bde. Bd. 2, Artikel Assassinier, (Archiv der europäischen Lexikographie, Abt. 1, Encyclopedien 32), Leipzig, Halle, Sp. 1894-1896.

\section{Sekundärliteratur}

Antos, Gerd / Wichter, Sigurd (Hrsg.) (2005), Wissenstransfer durch Sprache als gesellschaftliches Problem, (Transferwissenschaften 3), Frankfurt am Main, Wien.

Bourhis, Richard Y./ Maass, Anne (2005), „Linguistic Prejustice and Stereotypes / Linguistisches Vorurteil und Stereotyp“, in: Ulrich Ammon / Norbert Dittmar / Klaus J. Mattheier / Peter Trudgill (Hrsg.), Soziolinguistik. Ein internationales Handbuch zur Wissenschaft von Sprache und Gesellschaft. 2., vollständig neu bearb. und erw. Auflage. Teilbd. 2, (HSK 3), Berlin, New York, $1587-1601$.

Busch, Albert / Stenschke Oliver (Hrsg.) (2004), Wissenstransfer und gesellschaftliche Kommunikation. Festschrift für Sigurd Wichter zum 60. Geburtstag, Frankfurt am Main.

Eis, Gerhard (1965), Vor und nach Paracelsus. Untersuchungen über Hohenheims Traditionsverbundenheit und Nachrichten über seine Anhänger, (Medizin in Geschichte und Kultur 8), Stuttgart.

Foucault, Michel (2005), Die Heterotopien - Der utopische Körper. Zwei Radiovorträge, Frankfurt am Main.

Georges, Karl Ernst (1913), Ausführliches Lateinisch-Deutsches Handwörterbuch, 2 Bde., Bd. 1, Hannover, Leipzig.

Lasagabaster, David (2004), „Attitude / Einstellung“, in: Ulrich Ammon / Norbert Dittmar / Klaus J. Mattheier / Peter Trudgill (Hrsg.), Soziolinguistik. Ein internationales Handbuch zur Wissenschaft von Sprache und Gesellschaft. 2., vollständig neu bearb. und erw. Auflage. Teilbd. 1, (HSK 3), Berlin, New York, 399-405.

Lewis, Bernard (2001), Die Assassinen. Zur Tradition des religiösen Mordes im radikalen Islam, (Die andere Bibliothek 59), Frankfurt am Main.

Löffler, Heinrich (2010), Germanistische Soziolinguistik. 4., neu bearb. Auflage, (Grundlagen der Germanistik 28), Berlin.

Maalouf, Amin (2008), Der Heilige Krieg der Barbaren. Die Kreuzzüge aus Sicht der Araber, 5. Auflage, München.

Quasthoff, Uta (1973), Soziales Vorurteil und Kommunikation. Eine sprachwissenschaftliche Analyse des Stereotyps. Ein interdisziplinärer Versuch im Bereich von Linguistik, Sozialwissenschaft und Psychologie, (Fischer-Athenäum-Taschenbücher 2025), Frankfurt am Main.

Schjerve-Rindler, Rosita (2004), „Minderheit / Minority“, in: Ulrich Ammon / Norbert Dittmar / Klaus J. Mattheier / Peter Trudgill (Hrsg.), Soziolinguistik. Ein internationales Handbuch zur Wissenschaft von Sprache und Gesellschaft. 2., vollständig neu bearb. und erw. Auflage. Teilbd. 1, (HSK 3), Berlin, New York, 480-486. 
Stenschke, Oliver / Wichter Sigurd (Hrsg.) (2009), Wissenstransfer und Diskurs. Frankfurt am Main.

Strasser, Hermann / Brömme Norbert (2004), „Prestige und Stigma / Prestige and Stigma“, in: Ulrich Ammon / Norbert Dittmar / Klaus J. Mattheier / Peter Trudgill (Hrsg.), Soziolinguistik. Ein internationales Handbuch zur Wissenschaft von Sprache und Gesellschaft. 2., vollständig neu bearb. und erw. Auflage. Teilbd. 1, (HSK 3), Berlin, New York, 412-417.

Warnke, Ingo / Jürgen Spitzmüller (2008), „Methoden und Methodologie der Diskurslinguistik Grundlagen und Verfahren einer Sprachwissenschaft jenseits textueller Grenzen“, in: Ingo Warnke / Jürgen Spitzmüller (Hrsg.), Methoden der Diskurslinguistik. Sprachwissenschaftliche Zugänge zur transtextuellen Ebene, (Linguistik - Impulse \& Tendenzen 31) Berlin, New York, 3-56.

Wichter, Sigurd / Antos, Gerd (Hrsg.) (2001), Wissenstransfer zwischen Experten und Laien: Umriss einer Transferwissenschaft, Frankfurt am Main. 